\title{
NEW POSSIBILITIES FOR WELD ASSESSMENT USING INVERSE WAVEFIELD EXTRAPOLATION
}

\author{
Dr. Ir. C.H.P. Wassink ${ }^{1}$, Ir. K. Chougrani ${ }^{2}$ and J van der Ent ${ }^{3}$ \\ ${ }^{1}$ Applus RTD corporate, Capelle a/d IJssel, The Netherlands \\ ${ }^{2}$ Applus RTD Technological Center, Rotterdam, The Netherlands \\ ${ }^{3}$ Applus RTD Pipeline and Projects International, Rotterdam, The Netherlands
}

\begin{abstract}
New technological capabilities in both girth weld Non Destructive Testing (NDT) and girth weld material characterization open the door for new ways of weld assessment. The paper gives examples of new technologies currently being developed in NDT and investigates how these technologies could add value, in the field, on weld testing and assessment. The guiding principle in this investigation is that the data gathered in NDT should be sufficient for making an engineering decision. It will be argued that this is not currently the case as NDT is now used in a qualitative check on the welding process rather than a quantitative information source on the weld strength.
\end{abstract}

An overview of technologies like Inverse Wave Extrapolation (IWEX), Full Matrix Capture (FMC), and Sampling Phased Array (SPA) will be given. These technologies show promise to increase the quality of data received from Automated Ultrasonic Testing (AUT) of pipeline girth weld. The most important improvement is that an image of the defect is generated, in three dimensions, which allows for improved assessment of the impact of detected flaws on the weld strength. The paper will then show how these new capabilities could be combined with new technology in material testing and finite element calculations, which has specific value for strain based pipeline designs. The result is a vision on the future of girth weld assessment.

Keywords: NDT, IWEX, Rayscan, Tomography, Weld assessment

\section{INTRODUCTION}

In the last decade ultrasonic Phased Arrays have quickly started replacing older techniques in ultrasonic testing. It is expected that in this decade the transition to array transducers will be complete. Research into advanced imaging, using array transducers, shows that this is not the end point of the technology. Imaging technologies like IWEX, SPA and FMC have a potential to make testing much more reliable and to collect much more information on the girth weld. These technologies combine the ultrasonic signals received at many locations to create an image of the metal volume. The working principle is further explained in section 3.

The question could be raised what to do with all this information. Current zone based girth weld inspection systems, like the Rotoscan system, were not accepted by the market until the process of Engineering Critical Assessment (ECA) made it possible to make the added accuracy of that generation of testing technology productive [1]. This paper proposes a way to utilize the greater information content of IWEX results. As was the case with zone based inspections and ECA, we need to go to the engineering discipline using the NDT data to find out what this could be. This is an issue because NDT professionals generally have very little knowledge about the engineering decisions made based on the NDT data they collect. The technology described here is speculative, but the referred real life research will show that it may not be that far off.

\section{CURRENT WELD ASSESSMENT PRACTICE}

The strength of a weld is primarily determined by performing tensile and fracture toughness testing, performed on sample made using the welding procedure that will be used in the field. These tests are part of the welding procedure qualification that is required for every job, especially for pipelines. The primary function of NDT is to make certain that the actual weld quality in the field is similar enough to the quality of the welds used for material testing. The process flow, which for the purpose of this paper is called basic weld assessment, is shown in figure 1. 
With the advent of GMAW welding processes the type of flaws to be detected in pipelines changed. This led to a group of innovation that revolutionized the way girth welds were made. The innovation included the application of Automated Ultrasonic Testing (AUT) and Engineering Critical Assessment (ECA), mostly developed in the context of pipeline construction in Canada and offshore. Instead of rethinking the whole weld assessment process, these additional activities were bolted onto the basic weld assessment process. Added activities included stress analysis and additional materials testing for ECA, building of AUT calibration blocks and AUT qualification.

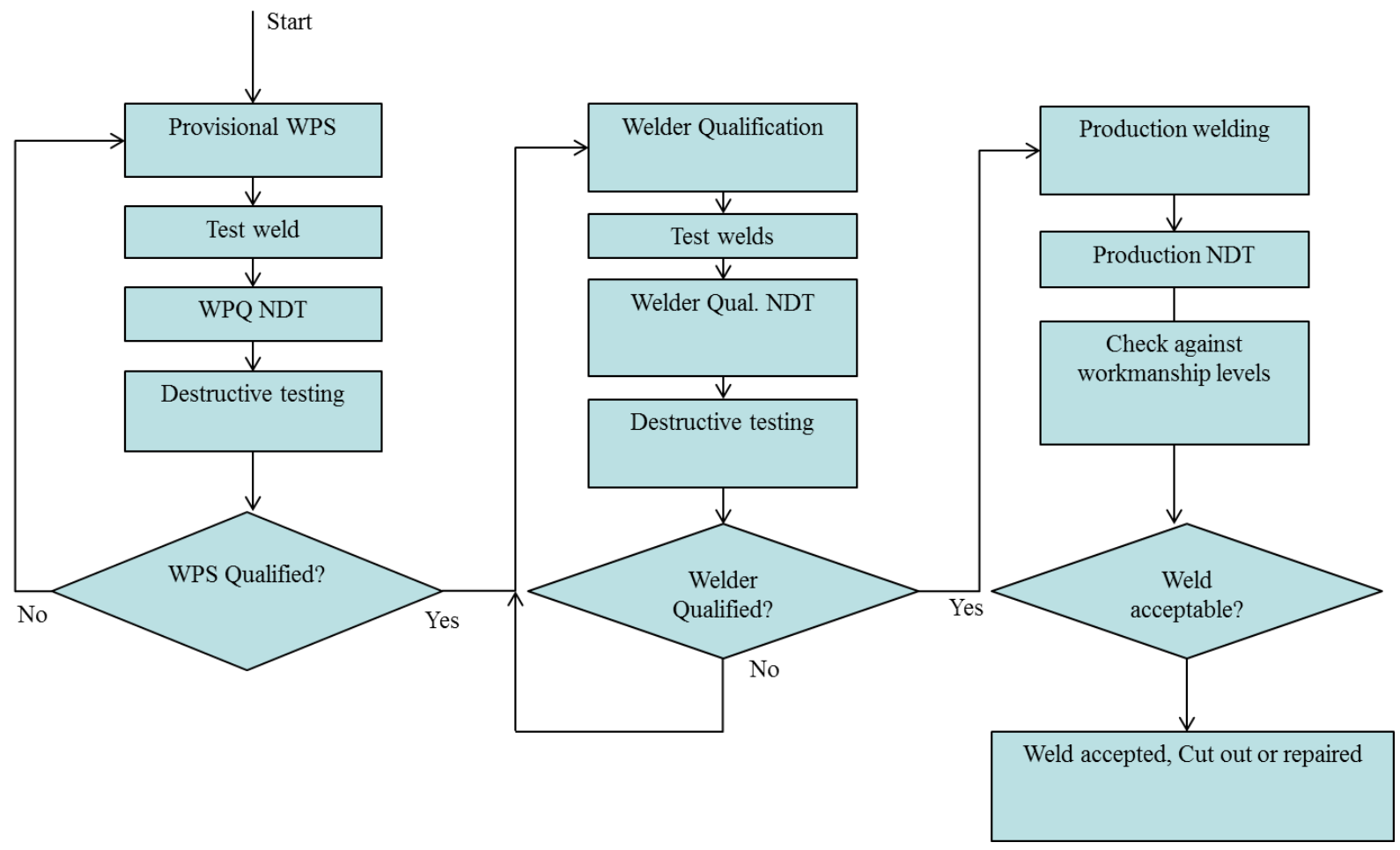

Figure 1 Current process for assessment of welds using workmanship standards

The order of activities gives rise to a number of issues, the main one being that performing enquiries into the stress requirements for the weld, after the welding procedure has already been qualified, seems to be the wrong order. In reality, these enquiries of course already happen before the welding procedure is qualified. In practice this means that many of the activities that are supposed to happen after the welding procedure has been qualified have to be done provisionally, as they are only valid when performed on weld produced with the qualified welding procedure.

\section{NEW POSSIBILITIES IN WELD NDT AND WELD FRACTURE MECHANICS}

In the last few years a number of developments in NDT have emerged that are expected to have an impact on girth weld testing. The main unifying feature of these development is, that they promise to give a three dimensional reconstruction of weld flaws. The direction of development of these technologies is not hard to predict as these development lag behind similar development in the medical and geophysical fields. In these fields, the combination of many more sensors, multiple testing methods and the use of digital image enhancement is well under way. In NDT it is just starting. The good news about this is that the wheel does not need to be invented again. It just needs to be adjusted to a different surface.

In ultrasonic testing practice, conventional pulse-echo ultrasonic systems are rapidly being replaced by Phased Array (PA) systems. For girth weld testing this transition is expected to be complete in this decade. In these systems the array transducers are used in the same way as in conventional ultrasonic testing. Features like sector scans look very nice, but have not resulted in improvement of the inspection results for girth weld testing. The Probability Of Detection (POD) and sizing accuracy have remained the same (for girth weld testing), and no more information can be obtained about the defect than before. The advantage is merely that the systems have become a lot more flexible, as many transducers can be replaced by a single PA transducer.

Array transducers do however have other possibilities. Instead of simulating conventional probes, the transducers can be used by pulsing each element individually (one after another) and receiving with all other elements. The received signals are then processed into an image by (mathematically) back propagating the signal to the location where it originated from. In the scientific NDT field, this is commonly 
called Full Matrix Capture (FMC). Several systems have been presented in literature, including the SPA system of IZFP [2] and the IWEX system of Applus RTD [3]. Paul Wilcox devoted his key note presentation during QNDE 2012 to these developments [4]. The result of the inspection with FMC systems is presented as a cross section of the weld, a presentation which can be readily understood by a lay person, unlike current AUT system reports. Typical example of IWEX images are shown below. The results can also be presented as a three dimensional model of the defect, which has been shown to have remarkable similarity to the actual defect geometry as obtained with curved wide plate testing.

In radiographic testing a similar development is underway. In 2009, Applus RTD introduced the RTD Rayscan system, which for the purpose of comparison can be equated to the PA systems in ultrasonic testing. The inspection result is essentially the same as with conventional film radiography (Rayscan regularly exceeds image quality of film radiography), but no improvement in defect detection and characterization is expected. A next step will be to implement the planar tomography technology that was previously demonstrated in TomoCAR [5]. This will result in a similar three dimensional reconstruction of defects as obtained with IWEX.

So far, practical validation projects have been started with several pipeline operating companies for IWEX. Although results are preliminary, as it will take some time to collect a statistically relevant amount of testing data, IWEX outperforms PA AUT considerable on traditional performance metrics. The sizing accuracy of PA AUT is generally considered to be between $1 \mathrm{~mm}$ and $1.5 \mathrm{~mm}$ [6]. IWEX sizing accuracy with prototype systems is around half this value. However there are several other advantages:

\section{Better knowledge on flaw type}

IWEX has been shown to accurately measure flaw orientation [7]. This will aid in determining what type of flaw is actually present e.g. a lack of fusion defect will generally follow the boundary of welding passes or the geometry of the weld preparation. As defect assessment criteria derived from radiography assume that the flaw type is known, ultrasonic testing (where flaw type is generally not known) has so far been penalized. This penalty could be removed.

An example is porosity, for which the flaw type is hard to determine with PA AUT. Porosity is currently included in e.g. API 1104 appendix A with the acceptance criterions of radiography for this reason. As with IWEX the ligament around porosity could be imaged, new acceptance limits for porosity would be possible.
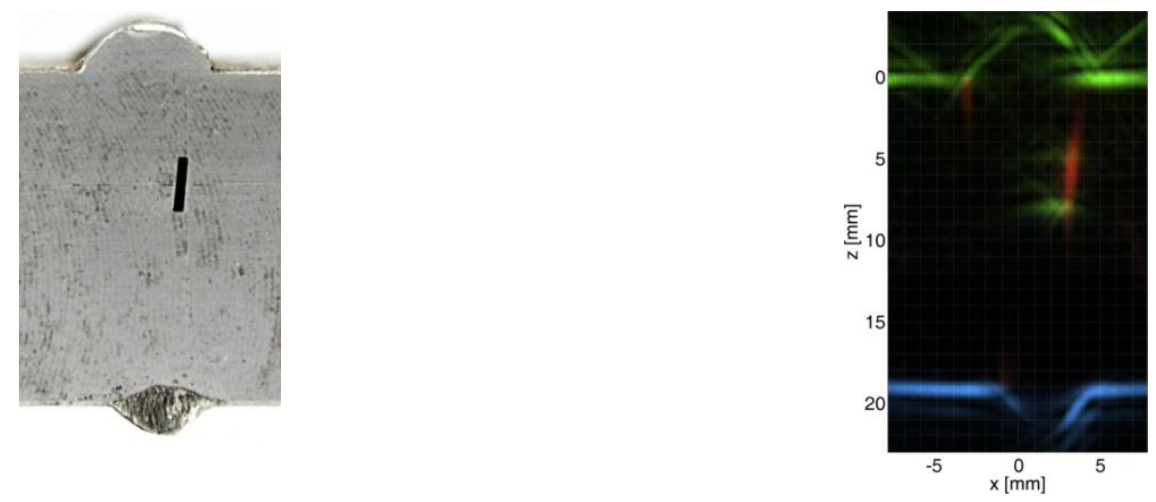

Figure 2 Metal cross section and IWEX image of an artificial flaw showing accurate representation of the size and orientation of the flaw. Also note the green signals at the tips of flaw which are used for sizing purposes. The capability can be used to determine the flaw type. 


\section{Measurement of pipe to pipe alignment / high-low}

IWEX results clearly show the pipe to pipe alignment. Pipe to pipe alignment is an important metric for strain based and deep water pipelines that has so far not been captured in NDT results.
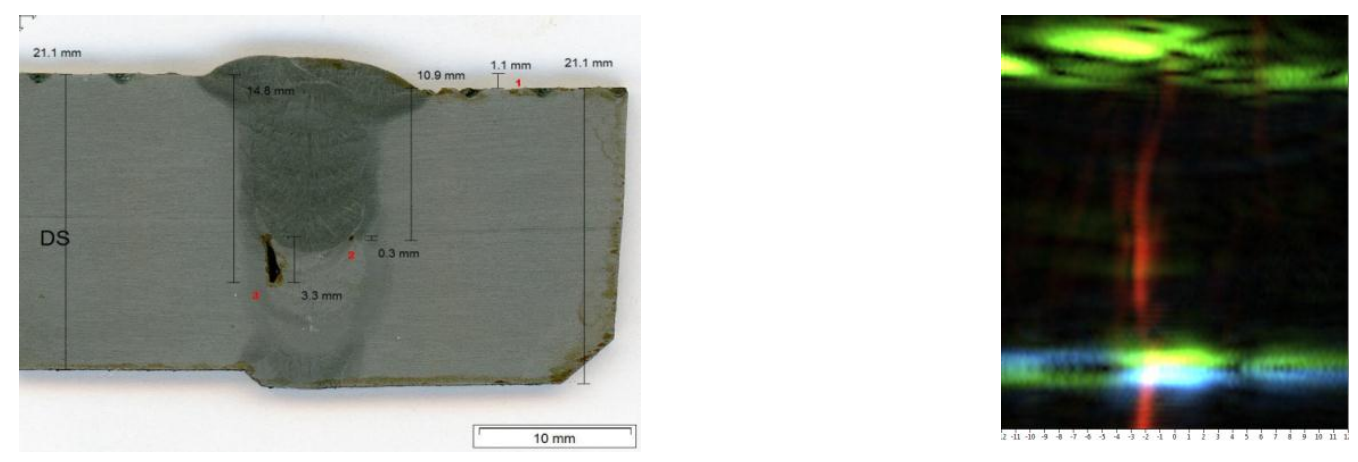

Figure 3 Macro sectioning and IWEX image of a weld showing a defect and high-low. The presence of high low in the image is clearly indicated by the green and blue signals on the ID. Notice how green, the ultrasonic signal coming from the left, and blue, the ultrasonic signal from the right, are flipped at weld centre

\section{Distinction between surface breaking and embedded defects}

IWEX results give a good indication of the ligament between a defect and the outer surfaces. So far, all defect close to the surface had to be evaluated as being surface breaking. Due to the limitation of PA AUT, close could mean a fair amount of the full metal thickness. Flaws will show up in multiple AUT zones due to the overlap of ultrasonic beams. This overlap is necessary to ensure full coverage of the weld. Flaws showing up in the first 2-3 zones to the surface might be surface breaking because of this.
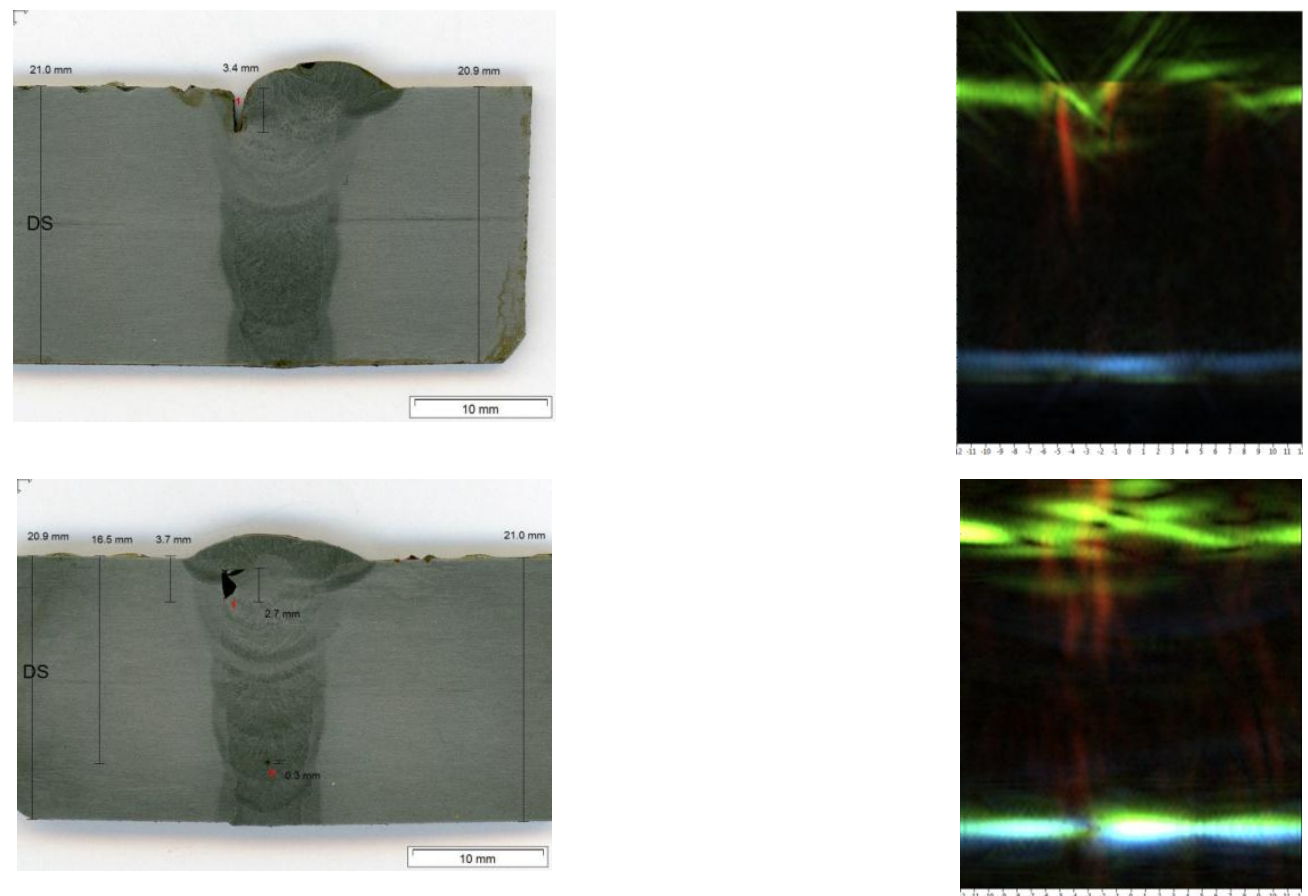

Figure 4 Macro sections and corresponding IWEX images of a surface breaking (top) and embedded (bottom) defect. Additional to these images the continuation of signals from the left and right side of the defect can be used to determine extension of flaws to the metal surface.

\section{Inspection result can be used as input to finite element models}

Due to the quality of the three dimensional reconstructions shown so far, with a technology that has not yet been optimised, it is expected to be feasible to input the results of IWEX into parametric finite element models. This would mean that the weld assessment could be based on meaningful quantities that are of interest to the pipeline fitness, instead of to intermediate results such as a failure assessment diagram (FAD). (See also picture on the next page) 


\section{A POSSIBLE FUTURE WELD ASSESSMENT PROCESS}

When considering these advantages, it is clear that IWEX gives an NDT result that is much better suited for making the engineering decisions on fitness of the weld. In fact, it could be argued that current NDT results are not suited at all for these decisions.

In this light, the advances in weld fracture mechanics should be mentioned. The agreement between models and results obtained from full scale pipeline testing and/or wide plate testing has progressed significantly [8], [9]. It is expected that prediction of e.g. strain capacity based on finite element models will achieve sufficient accuracy. The main limitation will be to achieve calculation times for a finite element model using IWEX results as the input, that are low enough for this assessment to be included in the production cycle time of girth welds.

When going this route, the other tests performed around the weld will also need to be adjusted. When making an engineering decision is used the guideline for what data should be obtained, it will be possible to determine which other tests should performed. Measuring remaining stress in the material and the hardness of the material is possible and these values may be needed as inputs for the finite element model.
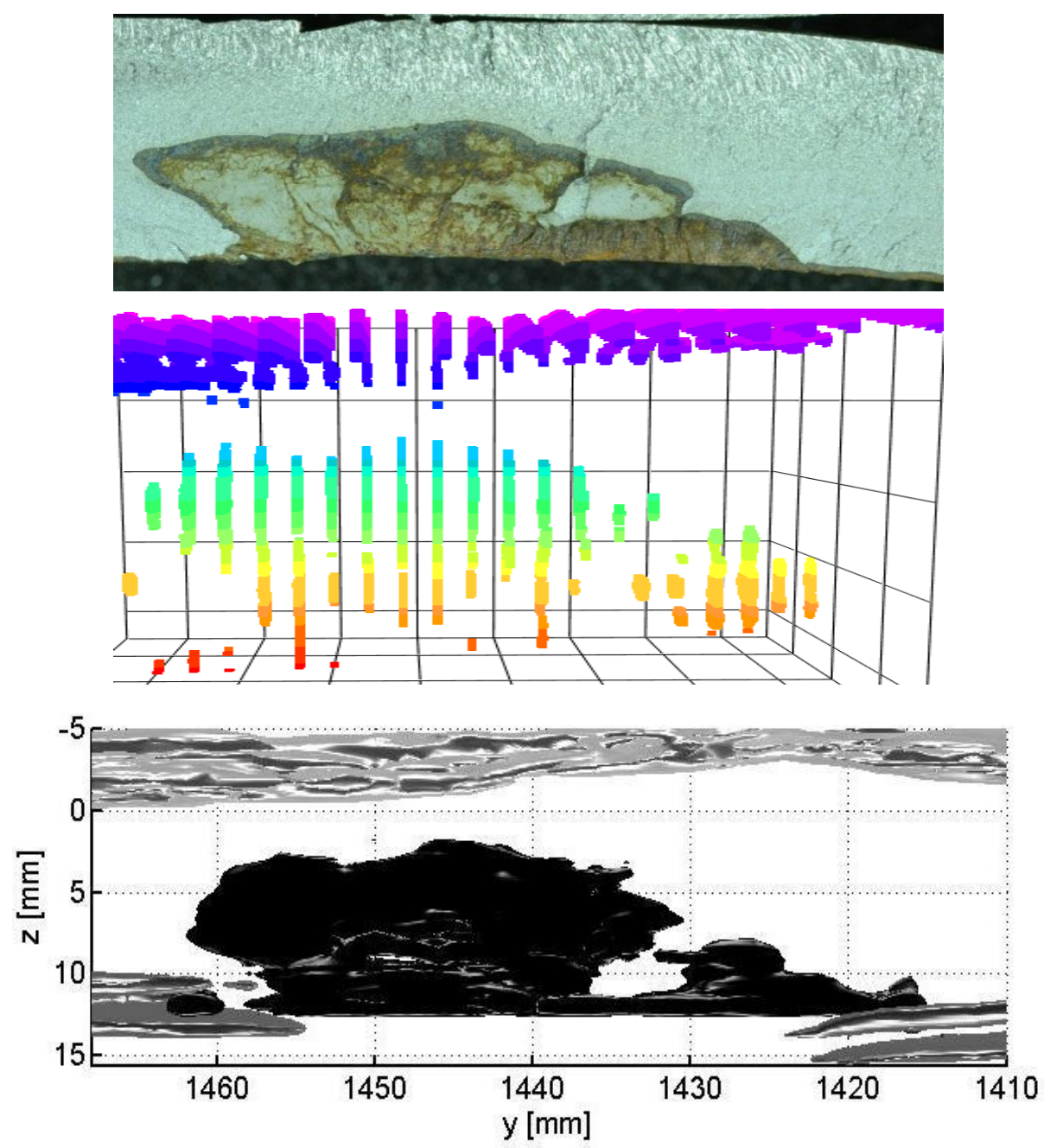

Figure 5 Metal cross section resulting for wide plates testing, 3D IWEX image and smoothed IWEX image. Clear correspondence of the three dimensional features of the defect are shown and could be used as input for FEM analysis.

When redesigning the weld assessment process, the requirement for the weld should be the starting point. Considering that stress based pipelines onshore have very different requirements for the fitness of the weld than strain based pipelines onshore or strain based pipelines offshore, this may lead to a dramatically different weld assessment process. A possible way to accommodate different requirements would be to introduce different application levels. At the lowest level NDT would just be used as a quality control on the welding process using traditional assessment methods. This would be a continuation of the current practice as depicted in figure 1. As newer ultrasonic methods clearly have a better performance that radiography, additional qualification requirements for ultrasonic testing above radiography should be dropped in these cases. 
The intermediate assessment level would involve cases where the quality of the weld itself needs to be assessed, as the weld itself is a critical feature. This would be a continuation of the current practice of ECA where the impact of detected flaws is evaluated using a failure assessment diagram.

At the highest assessment level, where a defect could potentially be an initiation for a fatigue defect or in case of strain based pipeline designs. In these cases the new UT method would be supplemented with measurement of additional (yet to be determined) measurements. These would then be the input for a finite element calculation that would result in an accurate prediction of e.g. the stress-strain curve of the flawed weld. At his highest level, the welding process would be developed after the assessment criteria are known.

\section{CONCLUSION}

New developments in NDT have a common feature that they move toward three dimensional reconstructions of flaw geometries. This is the case for both ultrasonic testing and radiography. When these methods are combined with advances in finite element modelling of flawed welds, a new assessment process of welds could be developed. In this process the start would be stress-strain requirements for the weld, and the NDT method combined with the finite element model would results in a prediction of the stress strain behaviour of the flawed weld, enabling a one-on-one comparison of requirement and resulting weld.

As stated, this is a speculative process, which' realisation is still years in the future, and require significant further development and validation. However all the component technologies have been developed and are available.

\section{ACKNOWLEDGEMENTS}

We want to acknowledge out partners at pipeline construction and pipeline owner companies for the trial projects that resulted in the images in this paper, and for the permission to use these images.

\section{REFERENCES}

[1] Wassink, C.H.P., Innovation in NDT, PhD Thesis, Delft University, 2012

[2] Bulavinov, A., Pinchuk, R., Pudovikov, S., Reddy, K.M., Walte, F. Industrial application of real-time 3D imaging by Sampling Phased Array, ECNDT 2010, Moscow, 2010.

[3] Chougrani, K., Portzgen N., IWEX: a new ultrasonic array technology for direct imaging of subsurface defects, WCNDT 2008, Shanghai, 2008

[4] Wilcox, P., Ultrasonic Arrays in NDE - Beyond the B-Scan, QNDE 2012, Denver, 2012

[5] Müller, J., Kiechle, H., Ewert, U., Rädel, C., Redmer, B., Mobile Computer Tomography for In-Service Inspection in Nuclear Power Plants, ECNDT 2006, Berlin, 2006

[6] BS 7910 Guide to methods for assessing the acceptability of flaws in metallic structures, Draft for public comment, London, 2012

[7] Deleye, X., Horchens, L., Chougrani, K., Experimental comparison of wave-field based ultrasonic imaging with other advanced ultrasonic weld inspection techniques, WCNDT 2012, Durban, 2012

[8] Hertelé, S., Coupled Experimental-Numerical Framework for the Assessment of Strain Capacity of Flawed Girth Welds in Pipeline, PhD Thesis, Gent University, 2012

[9] Wang, X., Kibey, S., Tang, H., Cheng, W., Minnaar K., Macia, M.L., Kan, W.C., Ford S.C., Newbury, B., Strain-based Design-Advances in Prediction Methods of Tensile Strain Capacity, International Journal of Offshore and Polar Engineering, Vol. 21, No. 1, pp. 1-7, 2011 